\title{
Spectroscopic Study of the Effects of Bioprotectant Systems on the Protein Stability
}

\author{
S. Magazù ${ }^{*}, 1$, F. Migliardo ${ }^{1,2}$, A.J. Ramirez-Cuesta ${ }^{3}$ and M.T.F. Telling ${ }^{3}$ \\ ${ }^{I}$ Dipartimento di Fisica, Università di Messina, S. Sperone C.da Papardo, P.O.Box 55 - 98166 Messina, Italy \\ ${ }^{2}$ Laboratoire de Dynamique et Structure des Matériaux Moléculaires, University of Lille 1, UMR CNRS 8024 - 59655 \\ Villeneuve d'Ascq CEDEX, France \\ ${ }^{3}$ ISIS Facility, Rutherford Appleton Laboratory, Chilton, Oxon, OX11 0QX, UK
}

\begin{abstract}
In the present article the effect of kosmotrope compounds, i.e. systems having the capability to stabilize biological macromolecules, is investigated by using complementary techniques. The attention is focused on the kosmotrope character of trehalose, a glucose disaccharide, compared to its homologous maltose and sucrose. Complementary techniques of neutron scattering, such as Inelastic Neutron Scattering (INS) and Quasi Elastic Neutron Scattering (QENS) allow to point out the capability of trehalose to strongly affect both the structural and dynamical properties of water. Finally the stabilization effect of trehalose on a well know protein, lysozyme, is studied as a function of temperature by Small Angle Neutron Scattering (SANS).
\end{abstract}

\section{INTRODUCTION}

It is well known that salts may stabilize protein promoting aggregation (Hofmeister effects) [1] and preferential hydration and increasing water surface tension [2], or can also influence protein stabilization by ion binding or screening of electrostatic (either attractive or repulsive) interaction [3]. It has been also suggested that salt-induced effects on the structure, stability and kinetics of protein are attributed to the binding of counterions, namely anions resulting in minimization of intramolecular electrostatic repulsion [3].

The analysis of thermodynamic parameters can offer some useful information on protein stabilization process. In fact, the variation of entropy of the aqueous solvent has a fundamental role in protein stability and folding. Cosolutes have a significant effect on the interplay between solvent entropy and protein stability [4], influencing chemical potential of both protein and solvent [5].

It has been demonstrated that the effectiveness of trehalose in protein stabilization is higher than that achieved using other sugars such as maltose, sucrose, glucose or fructose [6-8]. Some experimental findings obtained by several spectroscopic techniques indicate that the structural and the dynamical properties of water result perturbed by disaccharides, and in particular by trehalose [9-14]. The obtained findings are consistent with the picture of a disaccharide perturbing effect on the $\mathrm{H}_{2} \mathrm{O}$ tetrabonded network of water molecules [9-12]. Furthermore Elastic Neutron Scattering and viscosity measurements allowed for the "strongest" character in the Angell's classification

*Address correspondence to this author at the Dipartimento di Fisica, Università di Messina, P.O. Box 55, S.ta Sperone C.da Papardo, 98166 Messina, Italy; Tel: +390906765025; Fax: +39090395004;

E-mail: smagazu@unime.it scheme of trehalose in comparison with the other disaccharides to be pointed out [13].

In general cosolutes affect protein stability in different ways. Two of these are directly interaction with the protein (i.e. binding) and effects on the solvent (i.e. excluded volume effects) [15]. Organisms accumulate cosolutes as a physiological response to heat-shock, desiccation, osmotic shock and freezing.

Lysozyme is a well known protein responsible for breaking down the polysaccharide walls of many kinds of bacteria and thus it provides some protection against infection. From the applicative point of view, it is used as a preserving compound in many food products; the investigations on meat have showed that the addition of lysozyme resulted in a considerable inhibition of growth of the initial aerobic bacterial counts and a limitation of disadvantageous organoleptic changes during cold storage of samples [16]. The obtained results showed that lysozyme might be an effective agent extending shelf-life of portioned poultry meat. On the other hand, the scientific interest on lysozyme has stimulated extensive studies on the water arrangement around the protein, the interaction between lysozyme and salt molecules and structural and dynamical properties of lysozyme [17-21]. Sokolov and coworkers [19] have investigated the influence of glycerol and trehalose on lysozyme by Raman scattering, focusing particularly the attention on the low-frequency spectra. They conclude that protein is strongly coupled dynamically to trehalose and to glycerol and that glycerol provides superior suppression of protein dynamics than trehalose does at low temperature, while trehalose appears to be more effective at higher temperatures. A combined Raman and MD simulation study performed by Descamps and coworkers [20,21] on lysozyme/trehalose/H2O, lysozyme/maltose/H2O and lysozyme/sucrose/ $\mathrm{H} 2 \mathrm{O}$ solutions has been focused on the hydration properties of the protein in presence of disaccharides and their dependence on concentration, pointing out that trehalose hydrates preferentially 
lysozyme more than maltose and sucrose and confirming the higher destructuring effect.

The present work is focused on the use of complementary neutron scattering techniques to investigate the kosmotrope character of trehalose compared to its homologous maltose and sucrose, which posses the same chemical formula, but different structural and dynamical properties and then different bioprotective action. The influence of trehalose in lysozyme mixtures is finally described in the protein denaturation temperature range.

\section{MATERIALS AND METHODOLOGY}

Ultra pure powdered trehalose, maltose and sucrose, chicken-egg-white lysozyme and $\mathrm{H}_{2} \mathrm{O}$, purchased by Aldrich-Chemie.

INS measurements have been performed by using the indirect geometry time-of-flight spectrometer TOSCA at at ISIS, the world's leading pulsed neutron and muon source located at the Rutherford Appleton Laboratory (RAL, UK) [22]. The energy resolution of TOSCA is $\triangle \mathrm{E} / \mathrm{E} \approx 1.5-2 \%$ for energy transfers up to several hundred meV. This high resolution coupled with the high intensity of the ISIS source makes TOSCA ideal for studying the dynamics of water and water mixtures below $2000 \mathrm{~cm}^{-1}(250 \mathrm{meV})$ [22]. Measurements were performed at a temperature value of $27 \mathrm{~K}$ on hydrogenated trehalose, maltose and sucrose $\left(\mathrm{C}_{12} \mathrm{H}_{22} \mathrm{O}_{11}\right)$ in $\mathrm{H}_{2} \mathrm{O}$ at different weight fraction values corresponding to 2, 7, 10 and $14 \mathrm{H}_{2} \mathrm{O}$ molecules for each disaccharide molecule. The samples, contained in thin walled aluminium cells, were cooled to $27 \mathrm{~K}$ by a liquid helium cryostat. For all the investigated hydrogenated samples, the measurement time was 12 hours for each run. For the data treatment the standard GENIE programme has been used [22]. For all the plots the error bars to be considered are $\sim 5 \%$. The multiple scattering contribution has been minimised by using a thin sample in order to obtain a scattering transmission from the sample $\geq 90 \%$. The multiphonon neutron scattering contribution (MPNS), which can be significant at high temperature and large momentum transfer, has been calculated directly from the measured spectra by using a method of sequential iterations. Since measurements were performed at low temperature, the MPNS contribution is not large at the translational modes region (i. e. at low $\mathrm{Q}$ region) [23-25].

QENS measurements have been performed by using the IRIS high resolution spectrometer at ISIS (RAL, UK) $[26,27]$ in a temperature range of $283-320 \mathrm{~K}$ on hydrogenated trehalose, maltose and sucrose $\left(\mathrm{C}_{12} \mathrm{H}_{22} \mathrm{O}_{11}\right)$ in $\mathrm{H}_{2} \mathrm{O}$ and on partially deuterated trehalose, maltose and sucrose $\left(\mathrm{C}_{12} \mathrm{H}_{14} \mathrm{D}_{8} \mathrm{O}_{11}\right)$ in $\mathrm{D}_{2} \mathrm{O}$ at a weight fraction values corresponding to 19 water $\left(\mathrm{H}_{2} \mathrm{O}\right.$ and $\left.\mathrm{D}_{2} \mathrm{O}\right)$ molecules for each disaccharide molecule. In the deuterated solutions (at the investigated concentration) the coherent contribution to the total scattering cross section is $\sim 5 \%$. In the case of QENS in protonated samples, the attention is focused on the incoherent scattering arising from the self-correlation function which involves the motions of protons, the ratio between the incoherent cross-section $\sigma_{\mathrm{i}}$ and the scattering cross-section $\sigma_{\mathrm{s}}$ being $\sigma_{\mathrm{i}} / \sigma_{\mathrm{s}}=0.94$. We used the high resolution configuration of IRIS (graphite 002 and mica 006 analyser reflections) to measure sets of QENS spectra covering a Q, $\omega$-domain extending from $\hbar \omega=-0.3$ to $0.6 \mathrm{meV}$ (energy transfer) and $\mathrm{Q}=0.3$ to $1.8 \AA^{-1}$ (momentum transfer) $[26,27]$. The detectors used give a mean energy resolution of $\Gamma=8 \mu \mathrm{eV}$ of Half Width at Half Maximum (HWHM) as determined by reference to a standard vanadium plate. The sample was contained in an aluminium cell, that allows to obtain liquid samples in the form of slabs of $45 \times 35 \mathrm{~mm}^{2}$ and different thickness from 0.2 (for hydrogenated samples) to $1 \mathrm{~mm}$ (for deuterated samples). Using a sample changer accepting a vertical stack of three precisely aligned cuvettes it was possible to measure highly reliable spectra, and to keep the signal from the empty container below $1 \%$ of that from samples. The raw spectra were corrected and normalised using the standard GENIE procedures and the IRIS data analysis package [26,27].

SANS measurements have been performed by using the LOQ spectrometer at ISIS (RAL, UK) on lysozyme/ $\mathrm{D}_{2} \mathrm{O}$ mixtures and lysozyme $/ \mathrm{D}_{2} \mathrm{O} /$ trehalose mixtures for temperature values of $\mathrm{T}=310 \mathrm{~K}$ and $\mathrm{T}=333 \mathrm{~K}$. The Q-range covered by the LOQ spectrometer is from $0.007 \AA^{-1}$ to 0.287 $\mathrm{nm}^{-1}$. Incoming neutrons are monochromatized by a mechanical velocity selector with variable wavelength from $0.38 \mathrm{~nm}$ to $3 \mathrm{~nm}$, the wavelength resolution being $8 \%<\Delta \lambda / \lambda<18 \%$ (FWHM). The concentration of lysozyme was $10 \mathrm{mg} / \mathrm{ml}$. The lysozyme:trehalose mixtures were investigated at a mass ratio of 1:1. Lysozyme was dissolved in $40 \mathrm{mM} \mathrm{Na}$ acetate buffer at $\mathrm{pH} 4.25$ in $\mathrm{D}_{2} \mathrm{O}$. Measurements have been performed for five different contrast values $\left(20 \div 80 \% \mathrm{D}_{2} \mathrm{O} / 80 \div 20 \% \mathrm{H}_{2} \mathrm{O}\right.$ and $\left.100 \% \mathrm{D}_{2} \mathrm{O}\right)$. As an example in this paper we show results relative to $100 \% \mathrm{D}_{2} \mathrm{O}$ solutions. The contrast variation technique collecting data at different $\mathrm{D}_{2} \mathrm{O} / \mathrm{H}_{2} \mathrm{O}$ molar ratio has been employed in order to determine the protein scattering density length. In fact, the scattering length density of the aqueous solvent can be varied linearly from $-0.56 \cdot 10^{10}$ to $6.34 \cdot 10^{10} \mathrm{~cm}^{-2}$ by simply changing the $\mathrm{D}_{2} \mathrm{O} / \mathrm{H}_{2} \mathrm{O}$ molar ratio, according to the formula $\rho_{0}=[-0.56+6.90 \mathrm{R}] \cdot 10^{10} \mathrm{~cm}^{-2}$. Taking into account that the amount of labile hydrogen atoms in lysozyme in the native state is 193 and that the scattering length $b$ in $\mathrm{D}_{2} \mathrm{O}$ is $5.42 \cdot 10^{-3} \mathrm{~nm}$, the obtained $\rho$ values are $\rho=0.39 \cdot 10^{10} \mathrm{~cm}^{-2}$ at $\mathrm{T}=310 \mathrm{~K}$ and $\rho=0.29 \cdot 10^{10} \mathrm{~cm}^{-2}$ at $\mathrm{T}=333 \mathrm{~K}$. Corrections for inelastic effects, multiple scattering, absorption, sample container and background have been taken into account. For the analysis, the COLETTE software package has been used [28].

\section{RESULTS}

It is known that in the ice $0 \div 400 \mathrm{~cm}^{-1}$ spectrum two bands are observed at low energy: the first one, having a sharp peak at $\sim 56 \mathrm{~cm}^{-1}$, is dominant on the second one centred at $\sim 148 \mathrm{~cm}^{-1}$. They can be assigned to acoustic modes: in fact, that in the ice spectrum the peak at $\sim 56 \mathrm{~cm}^{-1}$ denotes the first Van Hove singularity in the dynamics of acoustic phonons. The two peaks present at $\sim 224 \mathrm{~cm}^{-1}$ and at $\sim 304 \mathrm{~cm}^{-1}$ with cutoffs on their right-hand sides are due to molecular optical modes [23-25].

Fig. (1) shows the INS spectra of disaccharides $/ \mathrm{H}_{2} \mathrm{O}$ mixtures at different concentration values in the $0 \div 400 \mathrm{~cm}^{-1}$ translational spectral region. It is observed from Fig. (1) that in presence of trehalose and sucrose the sharp peak of the low energy acoustic modes appears significantly lower and 
(a)

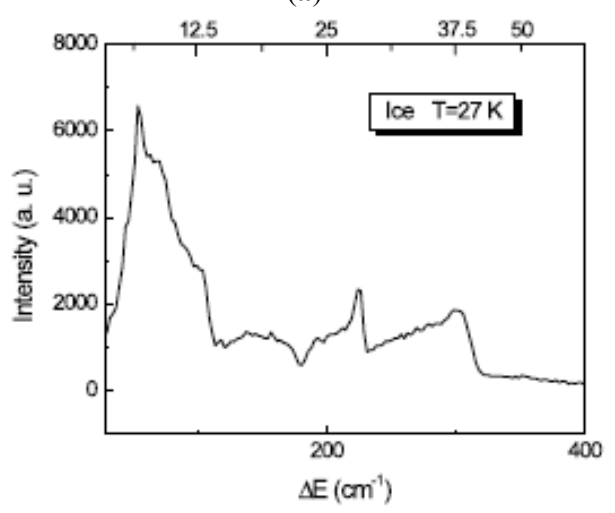

(b)

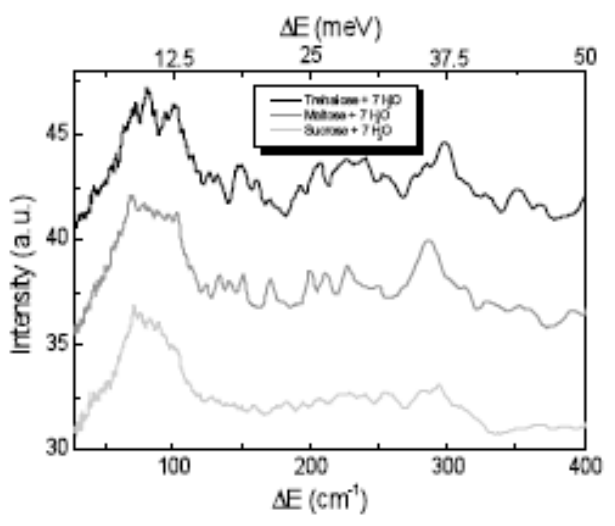

(c)

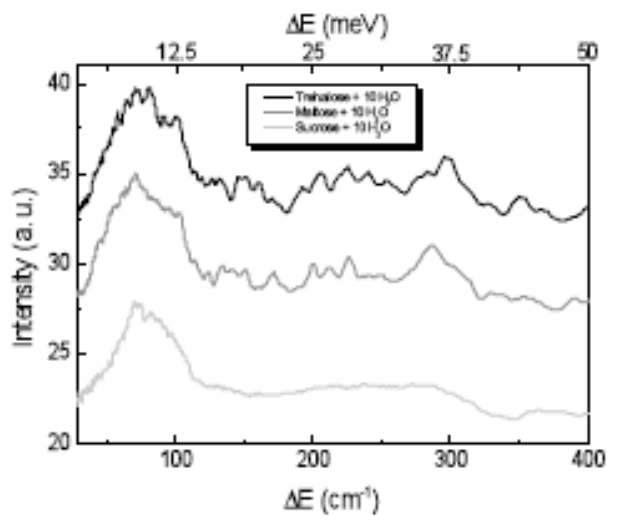

(d)

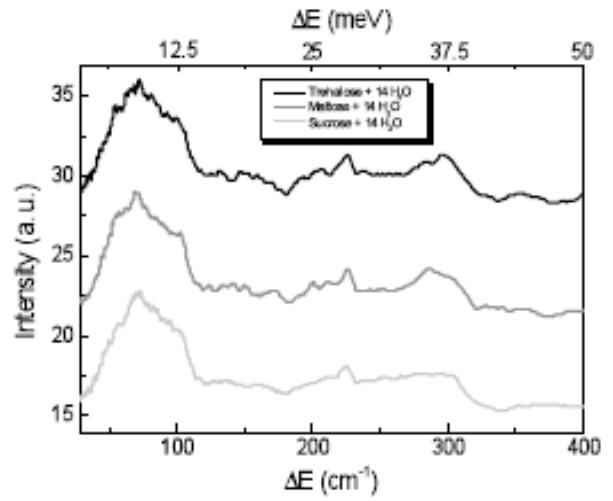

Fig. (1). INS spectra of (a) $\mathrm{H}_{2} \mathrm{O}$, (b) disaccharides/7 $\mathrm{H}_{2} \mathrm{O}$ mixtures, (c) disaccharides $/ 10 \quad \mathrm{H}_{2} \mathrm{O}$ mixture and (d) disaccharides/14 $\mathrm{H}_{2} \mathrm{O}$ mixture at $\mathrm{T}=27 \mathrm{~K}$ in the $0 \div 400 \mathrm{~cm}^{-1}$ translational spectral region. broader and shifted at $72 \mathrm{~cm}^{-1}$. The shift at higher energy is indicative of a strong interaction between disaccharides and water molecules. Important changes are also appreciable for the other bands and the second peak of the optical mode is present but deformed. The water contribution in this region starts to become marked only for the concentration value corresponding to $14 \mathrm{H}_{2} \mathrm{O}$ molecules for each disaccharide molecule, showing a spectrum more similar to that of water. Comparing trehalose and sucrose spectra, it is evident that sucrose is more strongly influenced by water and for sucrose mixtures the spectral features relative to the disaccharide contribution are more rapidly lost that for trehalose mixtures.

In the ice $400 \div 1060 \mathrm{~cm}^{-1}$ region it was found that the characteristic value for the librational band in the INS spectrum for different ice forms is the position of its lowenergy cut-off [23-25]. The observed shifts of the cut-off position are proportional to the transverse forces between the water molecules which are of different intensity for the different ice forms [23-25]. In order to point out the differences due to the trehalose, maltose and sucrose mixtures in the librational spectral region, in Fig. (2) the INS spectra of disaccharides $/ \mathrm{H}_{2} \mathrm{O}$ mixtures at different concentration values in this region are shown. A depression of the intensity of the cut-off is observed and, as it can be expected, it is more evident for trehalose, maltose and sucrose $/ 7 \mathrm{H}_{2} \mathrm{O}$, the cut-off becoming sharper by increasing the water content. Concerning the shift of the cut-off position, which for $\mathrm{H}_{2} \mathrm{O}$ is at $\sim 550 \mathrm{~cm}^{-1}$ [23-25], for all the investigated concentration values in trehalose mixtures we observe the same shift of $\sim 16 \mathrm{~cm}^{-1}$, whereas for maltose mixtures the shift is of $\sim 3 \mathrm{~cm}^{-1}$, and for sucrose mixtures no shift occurs.

The ice $1060 \div 1800 \mathrm{~cm}^{-1}$ spectral region corresponds to bending vibrational modes range [23-25]. As it can be observed by Fig. (3), which shows the INS spectra of disaccharides $/ \mathrm{H}_{2} \mathrm{O}$ mixtures at different concentration values in this region, the $\mathrm{H}_{2} \mathrm{O}$ spectrum is characterised by two distinct bands centred at $\sim 1224 \mathrm{~cm}^{-1}$ and $\sim 1608 \mathrm{~cm}^{-1}$, respectively. In the spectra of the investigated trehalose, maltose and sucrose $/ \mathrm{H}_{2} \mathrm{O}$ mixtures, these features are totally changed. The band at $\sim 1608 \mathrm{~cm}^{-1}$ is absent in the disaccharide $/ \mathrm{H}_{2} \mathrm{O}$ spectra, confirming that disaccharides are able to affect the water hydrogen bond $\mathrm{O}-\mathrm{H} \cdots \mathrm{O}$ bending modes connected to the strength and tetrahedrality of the hydrogen bonding [16-18]. In particular the role played by disaccharides is to impose to water a network which deviates from tetrahedral bonding and for which the hydrogen bonding among water molecules is diminished while that among disaccharides and water molecules is increased.

In QENS investigations of hydrogenated systems we can distinguish among different proton populations which contribute to the scattering law: i) protons of the disaccharide molecule and of its hydration shell, that follow the same diffusion law; ii) protons of the water molecules of higher hydration shells, whose diffusion is influenced by the presence of the disaccharide; iii) protons by bulk water that in our case are not present, as shown by ultrasonic, viscosity and light scattering measurements [9-12]. 
(a)

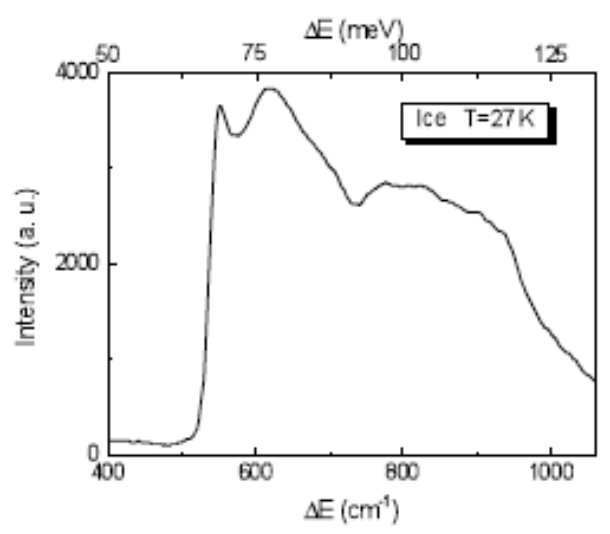

(b)

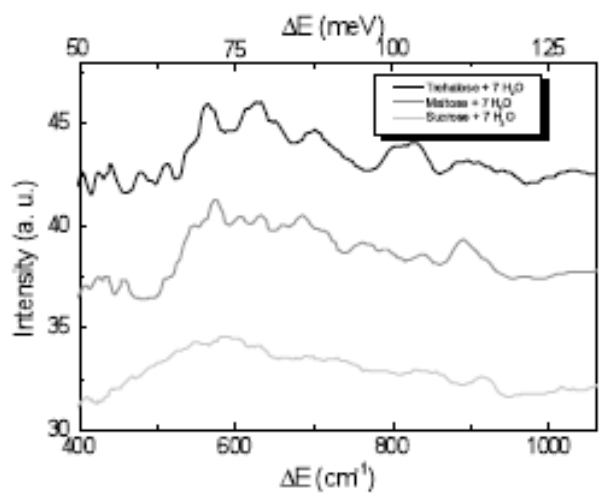

(c)

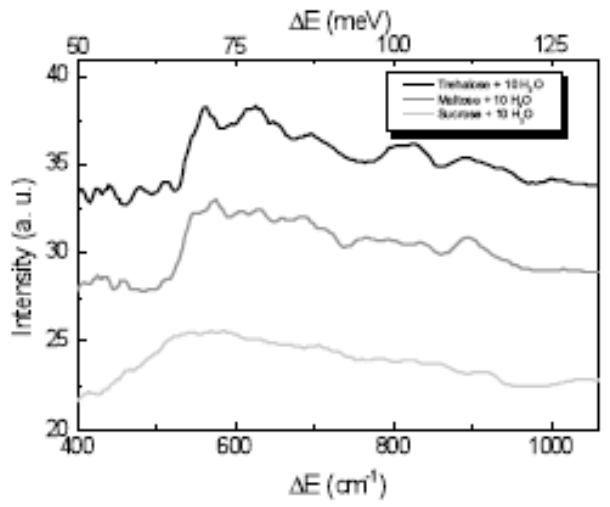

(d)

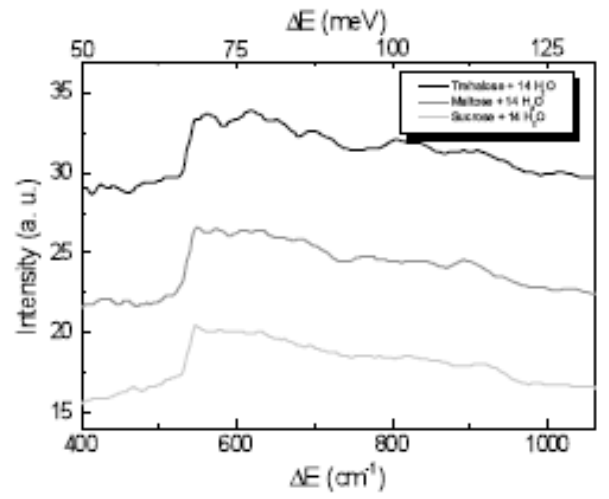

Fig. (2). INS spectra of (a) $\mathrm{H}_{2} \mathrm{O}$, (b) disaccharides $/ 7 \mathrm{H}_{2} \mathrm{O}$ mixtures, (c) disaccharides $/ 10 \quad \mathrm{H}_{2} \mathrm{O}$ mixture and (d) disaccharides/14 $\mathrm{H}_{2} \mathrm{O}$ mixture at $\mathrm{T}=27 \mathrm{~K}$ in the $400 \div 1060 \mathrm{~cm}^{-1}$ librational spectral region. (a)

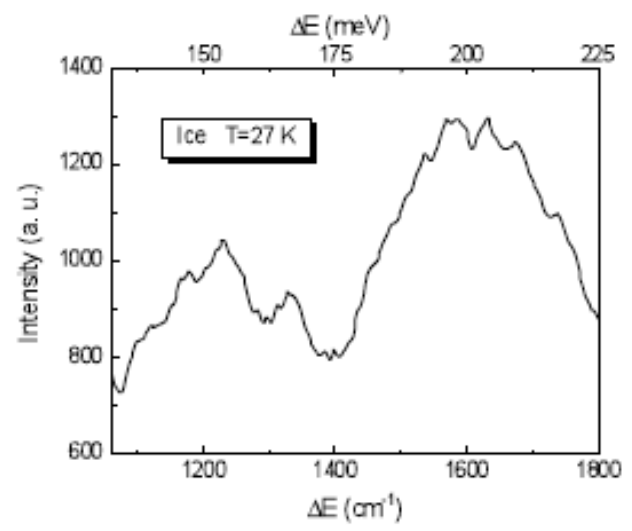

(b)

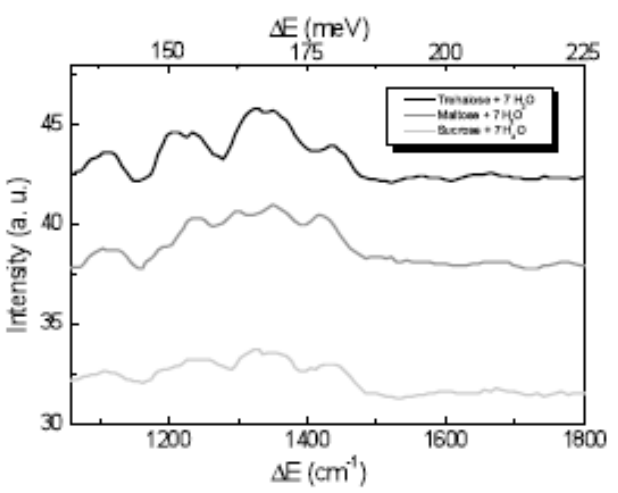

(c)

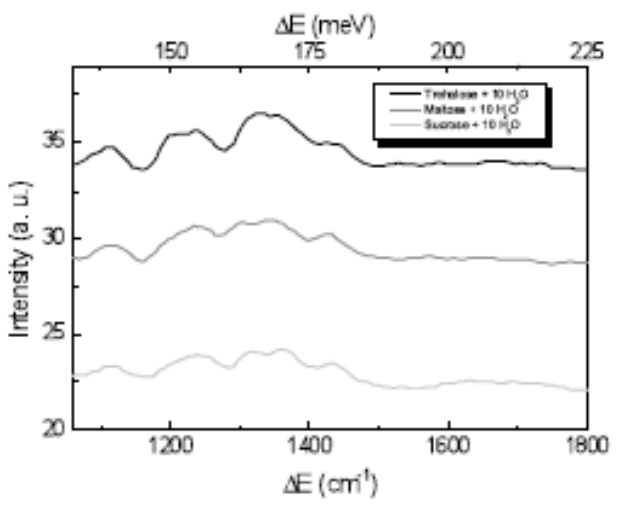

(d)

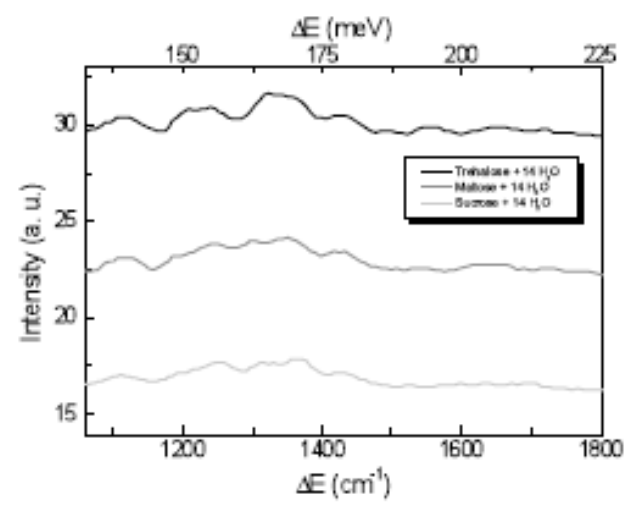

Fig. (3). INS spectra of (a) $\mathrm{H}_{2} \mathrm{O}$, (b) disaccharides/7 $\mathrm{H}_{2} \mathrm{O}$ mixtures, (c) disaccharides $/ 10 \quad \mathrm{H}_{2} \mathrm{O}$ mixture and (d) disaccharides $/ 14 \mathrm{H}_{2} \mathrm{O}$ mixture at $\mathrm{T}=27 \mathrm{~K}$ in the $1060 \div 1800 \mathrm{~cm}^{-1}$ bending spectral region. 
The spectra have been analyzed by using the fitting function:

$$
\begin{aligned}
& S_{\text {inc }}(Q, \omega)=A(Q)\left\{f_{\text {Disaccharide }}\left[F(Q) \frac{1}{\pi} \frac{\Gamma_{1}(Q)}{\Gamma_{1}^{2}(Q)+\omega^{2}}+(1-F(Q)) \frac{1}{\pi} \frac{\Gamma_{2}(Q)}{\Gamma_{2}^{2}(Q)+\omega^{2}}\right]\right. \\
& \left.+f_{\text {hydr }} \frac{1}{\pi} \frac{\Gamma_{3}(Q)}{\Gamma_{3}^{2}(Q)+\omega^{2}}\right\}
\end{aligned}
$$

where the first two terms refer to the translational and rotational contribution of hydrated disaccharide $\left(f_{\text {Disaccharide }}\right.$ and $f_{\text {hydr }}$ represent fraction factors of the total scattering from disaccharide and its strongly bonded water molecules), and the third one refers to hydration water $\left(f_{\text {Disaccharide }}+f_{\text {hydr }}=1\right)$. Therefore the dynamical information of the diffusive dynamics of disaccharide can be obtained by the analysis of disaccharide $+\mathrm{D}_{2} \mathrm{O}$ spectra analysis for which $\mathrm{f}_{\text {hydr }}$ results negligible.

For trehalose aqueous solutions, the $\mathrm{f}_{\text {hydr }}$ parameters keep constant with $\mathrm{Q}$ at the values of $0.032,0.108,0.223$ and 0.328 for $\mathrm{T}=283 \mathrm{~K}, 295 \mathrm{~K}, 308 \mathrm{~K}$ and $320 \mathrm{~K}$, respectively, corresponding to $18.0,15.7,12.2,9.0$ water molecules bound to the trehalose molecules. These hydration number values are in excellent agreement with those obtained by ultrasonic, hypersonic, viscosity and Raman scattering techniques [912]. As far as the hydration number values of the three disaccharides is concerned, at $\mathrm{T}=320 \mathrm{~K}$ the $\mathrm{f}_{\text {hydr }}$ parameters result $0.328,0.348,0.378$ for trehalose, maltose and sucrose solutions, respectively, corresponding to $9.0,8.4,7.5$ water molecules bound to the disaccharide molecules.

The linewidth $\Gamma_{3}$ of the translational contribution of water in presence of disaccharides and of pure water as a function of $\mathrm{Q}^{2}$ follows a Random Jump Diffusion (RJD) model [29], as shown in Fig. (4):

$\Gamma_{1}(\mathrm{Q})=\mathrm{D}_{\mathrm{s}} \mathrm{Q}^{2} /\left(1+\mathrm{D}_{\mathrm{s}} \mathrm{Q}^{2} \tau\right)$

where $D_{s}$ is the self diffusion coefficient of the molecule and $\tau$ is the residence time. The RJD model furnishes the diffusion coefficient $D_{s}$ value from the extrapolation to $Q \rightarrow 0$ and the residence time $\tau$ from the inverse of the asymptotic value at $\mathrm{Q} \rightarrow \infty$. Fig. (5) shows the obtained result from the fitting procedure for the diffusion coefficient and the residence time.

The whole water dynamics in trehalose solutions for $\mathrm{T}=283 \mathrm{~K}, 295 \mathrm{~K}, 308 \mathrm{~K}$ and $320 \mathrm{~K}$ resembles that of water at $256 \mathrm{~K}, 261 \mathrm{~K}, 263 \mathrm{~K}$ and $268 \mathrm{~K}$, indicating that the water has a diffusive behaviour strongly triggered by the trehalose molecules.

For the diffusion coefficient of water in the three disaccharide aqueous solutions we obtained at $\mathrm{T}=320 \mathrm{~K}$ the value of $D_{w}=8.31 \times 10^{-6} \mathrm{~cm}^{2} / \mathrm{s}$ for trehalose solution, $D_{w}=$ $8.46 \times 10^{-6} \mathrm{~cm}^{2} / \mathrm{s}$ for maltose solution and $\mathrm{D}_{\mathrm{w}}=8.60 \times 10^{-6}$ $\mathrm{cm}^{2} / \mathrm{s}$ for sucrose solution, with the values of residence times of $\tau=3.7 \mathrm{ps}, 3.4 \mathrm{ps}$ and $3.0 \mathrm{ps}$ for trehalose, maltose and sucrose solutions, respectively obtaining for the mean jump length $<\mathrm{l}>$ the value $<\mathrm{l}^{2}>^{1 / 2}=1.36 \AA, 1.31 \AA$ and $1.24 \AA$ for trehalose, maltose and sucrose solutions, respectively. In this case the water dynamics resembles that of water at $268 \mathrm{~K}$ in the case of trehalose solution, at $271 \mathrm{~K}$ in the case of maltose solution and at $277 \mathrm{~K}$ in the case of sucrose solution. Analogously to the trehalose aqueous solutions, all the disaccharides show a slowing down effect of the water dynamics, which is stronger for trehalose than the other disaccharides.

(a)

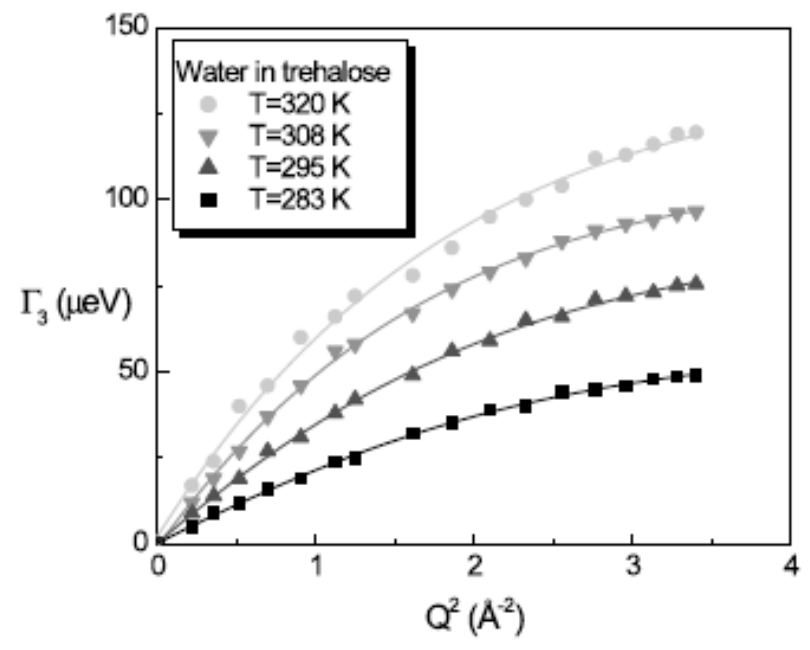

(b)

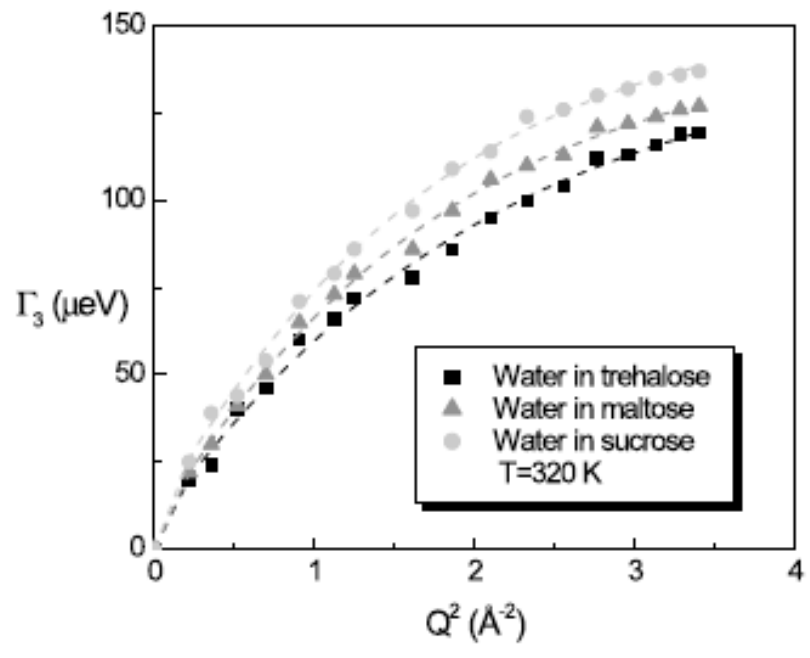

(c)

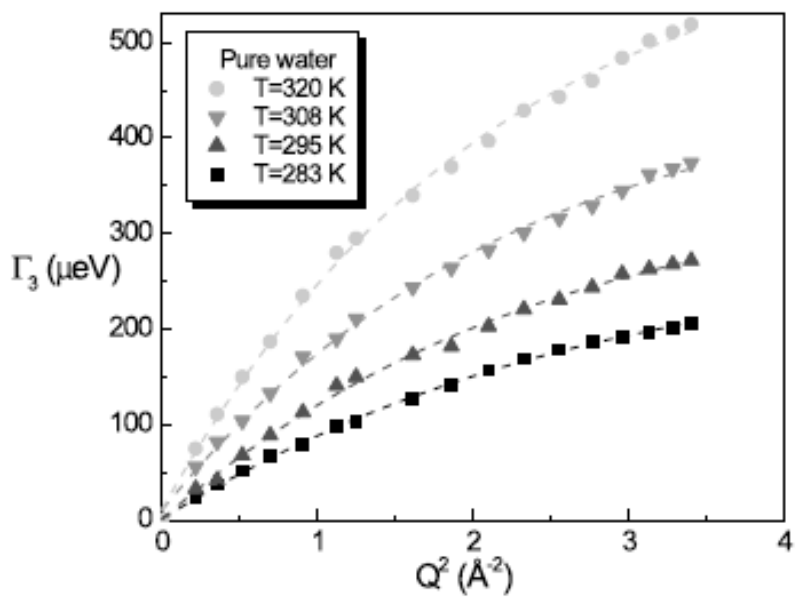

Fig. (4) Linewidth of the translational contribution as a function of $\mathrm{Q}^{2}$ for (a) water in trehalose aqueous solutions at four temperature values, (b) water in trehalose, maltose and sucrose aqueous solutions at $\mathrm{T}=320 \mathrm{~K}$, (c) for pure water for four temperature values. The solid lines are fits obtained following the RJD model (Eq. 2). 
(a)

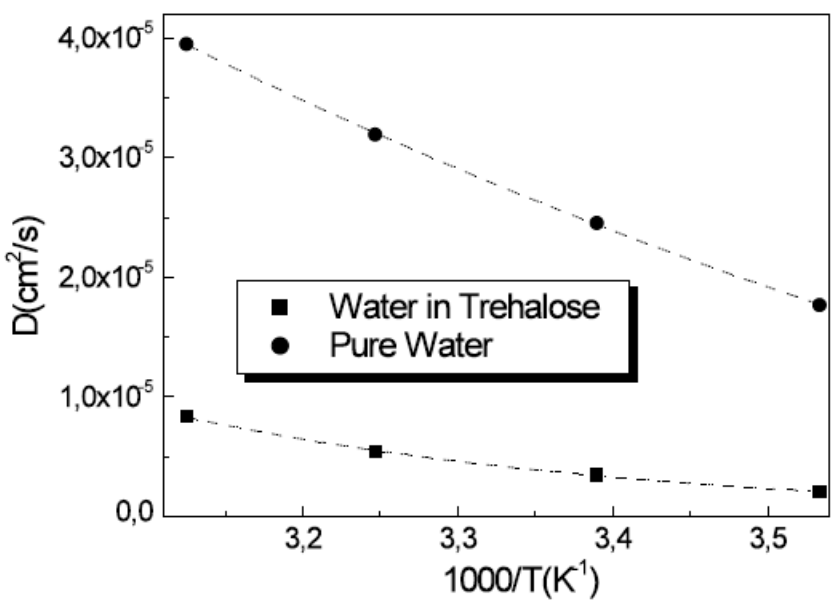

(b)

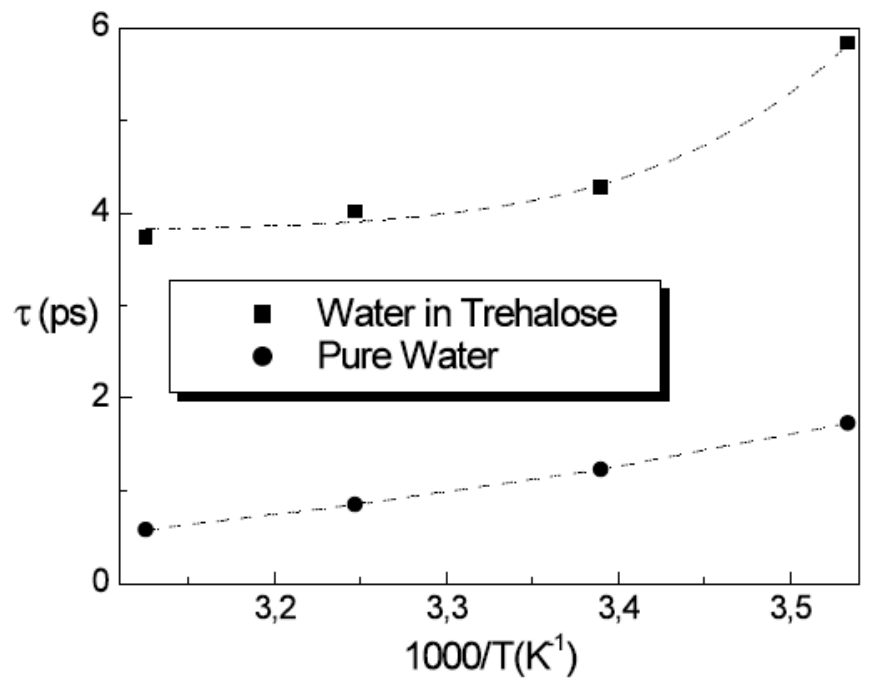

Fig. (5). (a) Diffusion coefficient and (b) residence time for water in trehalose solutions compared with pure water as a function of temperature.

In a SANS experiment, the scattering intensity is due to the interference of spherical waves originating from nuclei alike or different which are in the same (intraparticle contribution) or different molecules (interparticle contribution). The position of each nucleus can be written taking into account the centre of mass of the molecule $R_{n}$ and results $\underline{r}_{i(n)}=\underline{R}_{n}+\underline{R}_{i(n)}$. The coherent scattering cross-section in the "decoupling approximation" can be written as [30,31]:

$$
\left(\frac{\mathrm{d} \sigma}{\mathrm{d} \Omega}\right)_{\text {coh }}=N \Delta(\underline{Q})+N\left\langle\left|P_{i}(\underline{Q})\right|\right\rangle^{2} S(\underline{Q})
$$

where the term $\left\langle P[Q]^{2}\right\rangle$ is the form factor and contains information about the size, the shape and the internal structure of the molecule.

For rigorous monodispersed spheres $\left\langle P(\underline{Q})^{2}\right\rangle=\left\langle\left|P_{i}(\underline{Q})\right|\right\rangle^{2}=\Phi(\underline{Q})$ and the scattering cross section per unit volume becomes:

$\left(\frac{\mathrm{d} \sigma}{\mathrm{d} \Omega}\right)_{c o h}=N \Phi(\underline{Q}) S(\underline{Q})$
For $Q$ values smaller than the inverse of the characteristic dimensions of the particle, the spatial resolution is not sufficient to determine the shape, and only information about the size can be obtained by using the Guinier relation:

$\Phi(Q)=\Delta \rho^{2}\left(V^{\prime}\right)^{2} \exp \left(-\frac{Q^{2} R_{g}^{2}}{3}\right)$

where $V^{\prime}$ is the volume of the particle and $R_{g}$ is the gyration radius $[30,31]$.

In order to extract information about the size and the shape of lysozyme conformation, we have used two parallel procedures data analyses. The first one is based on a model fitting procedure that well approximates the lysozyme shape, the second one is based on the Guinier analysis procedure where the value of the gyration radius can be extracted by the gradient of the linear fitting function when the natural logarithm of the differential cross-section is reported versus $Q^{2}$ for small values of $Q^{2}$.

Fig. (6) shows the intensity profiles $I(Q)$ as a function of $\mathrm{Q}$ of lysozyme $/ \mathrm{D}_{2} \mathrm{O}$ solution for $\mathrm{T}=310 \mathrm{~K}$ and $\mathrm{T}=333 \mathrm{~K}$ together with the Guinier plot. Following the prolate ellipsoidal model, the form factor, normalized by the particle volume and averaged over all possible orientations of the ellipsoid, is expressed by:

$$
\left.P(Q)=\frac{\text { const. }}{V_{\text {ell }}}\left(\rho_{\text {ell }}-\rho_{\text {solv }}\right)^{2} \int_{0}^{1} f^{2}\left[Q r_{0}\left(1+x^{2}\right)\left(v^{2}-1\right)\right)^{1 / 2}\right] d x+B K G
$$

where $f(z)=3 V_{\text {ell }} \frac{(\sin z-z \cos z)}{z^{3}}, V_{\text {ell }}=(4 \pi / 3) r_{a} r_{b}^{2}$ and $v=\frac{r_{a}}{r_{b}}$.

It is evident that this model fits in a very good way the experimental data profiles. For the gyration radius, by using the formula $R_{g}{ }^{2}=1 / 5\left(2 r_{a}{ }^{2}+r_{b}{ }^{2}\right)$, we obtain the values of $\mathrm{R}_{\mathrm{g}}=11.8 \AA$ and $\mathrm{R}_{\mathrm{g}}=12.7 \AA$ for $\mathrm{T}=310 \mathrm{~K}$ and $\mathrm{T}=333 \mathrm{~K}$. The values relative to the two temperature values indicate an increase of the protein dimensions: this means that denaturation processes occurred in the investigated temperature range. On the other hand, the Guinier analysis procedure furnishes at $\mathrm{T}=310 \mathrm{~K}$ the value of $\mathrm{R}_{\mathrm{g}}=11.8 \AA$ and at $\mathrm{T}=333 \mathrm{~K}$ the value of $\mathrm{R}_{\mathrm{g}}=12.6 \AA$. The obtained absolute values are not significant due to the strong approximation in which the analysis procedure is valid. On the contrary, the obtained relative values, maintaining the analogous ratio shown by the model fitting procedure, are important because furnish the confirmation that lysozyme has lost its native conformation.

A comparison of lysozyme $/ \mathrm{D}_{2} \mathrm{O} /$ trehalose solutions spectra for $T=310 \mathrm{~K}$ and $\mathrm{T}=333 \mathrm{~K}$ is shown in Fig. (7). Since in this case we are not able to fit the intensity profile by means of a suitable model, numerical values of the lysozyme dimensions in solution in presence of trehalose are extracted by using the Guinier procedure, furnishing at $\mathrm{T}=310 \mathrm{~K}$ the value of $R_{g}=16.2 \AA$ and at $T=333 \mathrm{~K}$ the value of $R_{g}=16.4 \AA$. We observe that by increasing temperature the value of the gyration radius is not changed, this circumstance emphasising the stabilising effect of trehalose on lysozyme.

\section{DISCUSSION AND CONCLUSION}

In this paper a combined study performed by INS, QENS and SANS on binary disaccharide $/ \mathrm{H}_{2} \mathrm{O}$ mixtures and ternary 
(a)

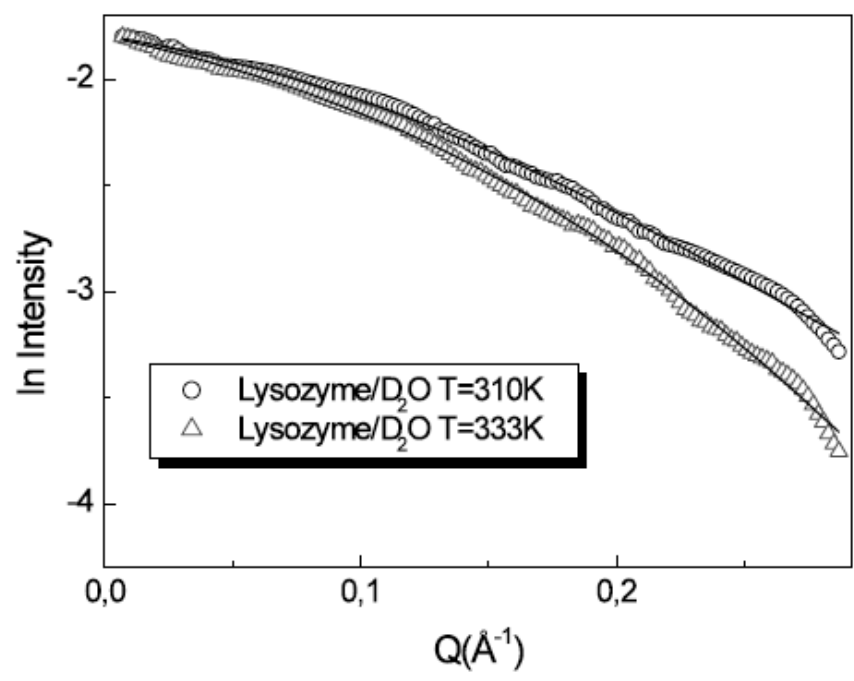

(b)

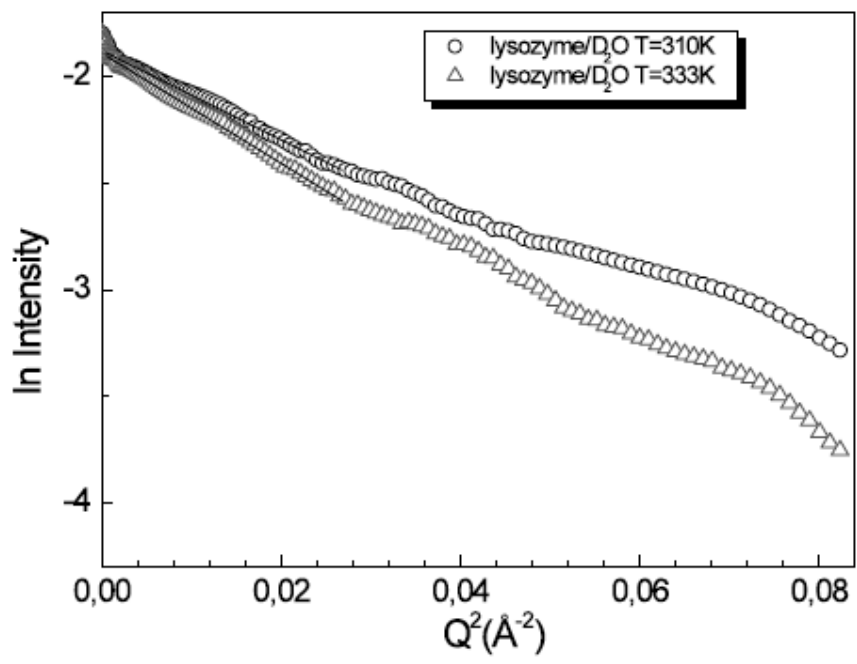

Fig. (6). (a) $\mathrm{I}(\mathrm{Q})$ as a function of $\mathrm{Q}$ and (b) Guinier plot of lysozyme $/ \mathrm{D}_{2} \mathrm{O}$ solution for $\mathrm{T}=310 \mathrm{~K}$ and $\mathrm{T}=333 \mathrm{~K}$.

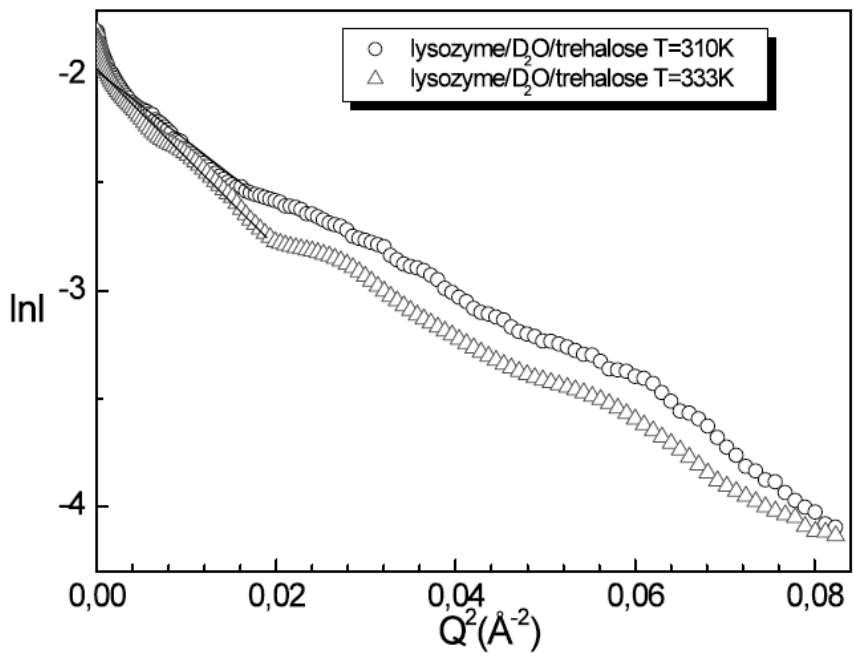

Fig. (7). Guinier plot of lysozyme $/ \mathrm{D}_{2} \mathrm{O} /$ trehalose solution for $\mathrm{T}=310 \mathrm{~K}$ and $\mathrm{T}=333 \mathrm{~K}$. disaccharide/protein $/ \mathrm{H}_{2} \mathrm{O}$ mixtures as a function of $\mathrm{Q}$, concentration and temperature is shown. The goal is to highlight the kosmotrope nature of trehalose and its capability to stabilize biological systems.

The physical picture obtained from the performed studies shows that the highest bioprotectant effectiveness of trehalose in comparison with the other disaccharides is due to the combined effect of different co-factors. What emerges is that the biological action of disaccharides can be explained by the strong interaction with water molecules, as emphasised by the INS findings. This capability imply that disaccharides, and in particular trehalose, promote an extensive layer of structured water around its neighbourhood, which destroys the tetrahedral H-bond network of pure water, providing a structure whose spatial positions and orientations are not compatible with those of ice. On the other hand, the QENS results point out that the disaccharides strongly affects also the water dynamics, with a higher effect in the case of trehalose water solutions. The conclusion is that trehalose, besides imposing an order on the tetrahedral network of water, significantly slow down the dynamics of water. The higher slowing down effect of the diffusive dynamics observed for trehalose is evidently linked to its extraordinary capability to "switch off" the metabolic functions. Therefore such a finding, implying for trehalose a higher kosmotrope character, can account for its bioprotective effectiveness. This aspect is emphasised by the SANS results, which show that the protein dimensions are not changed by increasing trehalose, therefore trehalose inhibits significantly the temperature induced swollening of lysozyme.

\section{ACKNOWLEDGEMENTS}

Federica Migliardo gratefully acknowledges UNESCOL'Oréal for the International Fellowship for Young Women in Life Sciences 2008. The authors gratefully acknowledge the ISIS facility (Chilton, UK), for the dedicated runs at the IRIS, TOSCA and LOQ spectrometers.

\section{REFERENCES}

[1] Baldwin RL. How Hofmeister ion interactions affect protein stability. Biophys J 1996; 71: 2056-63.

[2] Cacace MG, Landau EM, Ramsden JJ. The Hofmeister series: salt and solvent effects on interfacial phenomena. Quart Rev Biophys 1997; 30: 241-77.

[3] Record MTJ, Zhang W, Anderson CF. Analysis of effects of salts and uncharged solutes on protein and nucleic acid equilibria and processes: a practical guide to recognizing and interpreting polyelectric effects, Hofmeister effects and osmotic effects of salts. Adv Protein Chem 1998; 51: 281-353.

[4] Donna B, Wright DD, Banks JR, Lohman JL, Hilsenbeck L, Gloss M. The effect of salts on the activity and stability of Escherichia coli and haloferax volcanii dihydrofolate reductases. J Mol Biol 2002; 323: 327-44.

[5] Lee JC, Timasheff SN. The stabilization of proteins by sucrose. J Biol Chem 1981; 256: 7193-201.

[6] Sola-Penna M, Meyer-Fernandes JR. Protective role of trehalose in thermal denaturation of yeast Pyrophosphatase. Z Naturforsch 1994; 49C: 327-30.

[7] Sola-Penna M, Meyer-Fernandes JR. Trehalose protects yeast pyrophosphatase against structural and functional damage induced by guanidinium chloride. Z Naturforsch 1996; 51: 160-4.

[8] Sola-Penna M, Meyer-Fernandes JR. Stabilization against thermal inactivation Promoted by sugars on enzyme structure and function: Why is trehalose more effective than other sugars? Arch Biochem Biophys 1998; 360: 10-4. 
[9] Branca C, Magazù S, Maisano G, Migliardo P. Anomalous cryoprotective effectiveness of trehalose: raman scattering evidences. J Chem Phys 1999; 111: 281-7.

[10] Branca C, Faraone A, Magazù S, Maisano G, Migliardo F, Migliardo P, Villari V. Structural and dynamical properties of trehalose-water solutions: anomalous behaviour and molecular models. Rec Res Develop Phys Chem 1999; 3: 361-403.

[11] Branca C, Magazù S, Maisano G, Migliardo F, Romeo G. $\alpha, \alpha-$ trehalose/water solutions. 5. hydration and viscosity in dilute and semidilute disaccharide solutions. J Phys Chem B 2001; 105: 10140-5.

[12] Branca C, Magazù S, Maisano G, Migliardo F. New perspectives on bioprotectant complex molecules: spectroscopic findings. Rec Res Develop Phys Chem 2002; 6: 35-73.

[13] Magazù S, Maisano G, Migliardo F, Mondelli C. Mean square displacement relationship in bioprotectant systems by elastic neutron scattering. Biophys J 2004; 86: 3241-9.

[14] Magazù S, Migliardo F, Ramirez-Cuesta AJ. Inelastic neutron scattering study on bioprotectant systems. J R Soc Interf 2005; 2: $527-31$.

[15] Saunders AJ, Davis-Searles PR, Allen DL, Pielak GJ, Erie DA. Osmolyte-induced changes in protein conformational equilibria. Biopolymers 2000; 53: 293-307.

[16] Kijowski J, Marciszewska C, Cegielska-Radziejewska R. Quality and microbiological stability of chilled chicken breast muscles treated with a lysozyme solution. Polish J Food Nutr Sci 2002; 11: 47-54.

[17] Minezaki Y, Niimura N, Ataka M, Katsura T. Small angle neutron scattering from lysozyme solutions in unsaturated and supersaturated states (SANS from lysozyme solutions). Biophys Chem 1996; 58: 355-63.

[18] Svergun DI, Richard S, Koch MHJ, Sayers Z, Kuprin S, Zaccai, G. Protein hydration in solution: experimental observation by x-ray and neutron scattering. Proc Natl Acad Sci USA 1998; 95: 226772 .
[19] Caliskan G, Mechtani D, Roh JH, et al. Protein and solvent dynamics: How strongly are they coupled? J Chem Phys 2004; 121 : 1978-83.

[20] Hedoux A, Affouard F, Descamps M, Guinet Y, Paccou L. Microscopic description of protein thermostabilization mechanisms with disaccharides from Raman spectroscopy investigations. J Phys Condens Matter 2007; 19: 205142-8.

[21] Ionov R, Hedoux A, Guinet Y, et al. Sugar bioprotective effects on thermal denaturation of lysozyme: Insights from Raman scattering experiments and molecular dynamics simulation. J Non-Crystalline Solids 2006; 352: 4430-6.

[22] Colognesi D, Parker SF. The TOSCA Manual. Rutherford Appleton Laboratory UK 1999.

[23] Kolesnikov AI, Li JC, Ahmad NC, et al. Neutron spectroscopy of high-density amorphous ice. Physica B 1999; 263-264: 650-2.

[24] Kolesnikov AI, Li JC, Parker SF, Eccleston RS, Loong C-K. Vibrational dynamics of amorphous ice. Phys Rev B 1999; 59: 3569-78.

[25] Li JC. Inelastic neutron scattering studies of hydrogen bonding in ices. J Chem Phys 1996; 105: 6733-55.

[26] Adams MA, Howells WS, Telling MTF. The IRIS User Guide $2^{\text {nd }}$ Ed Rutherford Appleton Laboratory, UK 2001.

[27] Telling MTF, Howells WS, MODES - IRIS data analysis ( $1^{\text {si }}$ Edition), Rutherford Appleton Laboratory, UK 2003.

[28] King SM. Using Colette. Rutherford Appleton Laboratory UK 2004.

[29] Bee M. Hilger, A, Ed. Quasielastic Neutron Scattering. Bristol and Philadelphia 1988

[30] King, SM. Small angle neutron scattering, in: modern techniques for polymer characterisation. In: Pethrick RA, Dawkins JV, Eds USA: John Wiley \& Sons 1999; p. 171.

[31] Lindner P. Neutron, X-ray and Light Scattering, Th Zemb Holland 1991.

This is an open access article licensed under the terms of the Creative Commons Attribution Non-Commercial License (http://creativecommons.org/licenses/by$\mathrm{nc} / 3.0 /$ ) which permits unrestricted, non-commercial use, distribution and reproduction in any medium, provided the work is properly cited. 